\title{
Visualization of three dimensional earth fissures in geological structure
}

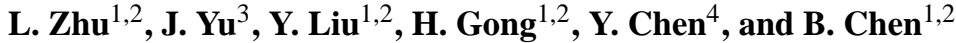 \\ ${ }^{1}$ College of Resources Environment and Tourism, Capital Normal University, Beijing, China \\ ${ }^{2}$ Laboratory Cultivation Base of environment process and digital simulation, Beijing, China \\ ${ }^{3}$ Key Laboratory of Earth Fissures Geological Disaster, Ministry of Land and Resources, Jiangsu, China \\ ${ }^{4}$ Land and Water Laboratory, Canberra, Australia
}

Correspondence to: L. Zhu (hi-zhulin@163.com)

Published: 12 November 2015

\begin{abstract}
This paper proposes a new method for visualizing the earth fissures of geological structure in three dimensional (3-D) domains on the basis of the seismic data and features information of earth fissures. The seismic data were interpreted for obtaining the stratagraphic data with various lithological information and the depth of the earth fissures. The spatial distribution of the ground fissures including the dip, strike and width were digitalized on an ArcGIS platform. Firstly, the 3-D geological structure was rebuilt using the Generalized Tri-Prism (GTP) method which is a real solid method for displaying geological structures. The GTP method can reflect the inner material of the strata and can simulate complicated geological structures such as faults and stratagraphic pinch outs. The upper and lower surfaces of each stratum consist of Triangle Irregular Networks (TIN). The inner solid between the two surfaces are a series of triangular prisms. Secondly, since the width of the ground fissure gradually decreases with depth, multiple edge lines of the earth fissures on the bottom stratum surface are deduced on the basis of the fissure characteristics. Then, the model of the earth fissures consisting of a series of triangular pyramids can be constructed using these points and the edge lines. A cutting operation was carried out on the 3-D geological structure using this ground fissures model. If the surfaces of the ground fissures model intersects with the GTPs in the geological structure model, new GTPs were generated within the local regions. During this process, the topological relations between TIN, triangular prism and lines were reconstructed so that the visualization of ground fissures in the geological structure model is realized. This method can facilitate the mechanism for studying fissures and avoid the gaps between the fissure solid and the geological structure to accurately reflect their 3-D characteristics.
\end{abstract}

\section{Introduction}

Earth fissures are induced by natural or man-made factors such as volcano, ice, water, and exploration of the subsurface resources (water, oil and gas). Inland areas, especially in many alluvial basins, earth fissures are mainly caused by tensional forces related to differential land subsidence, which is mainly related to long-term groundwater pumping in excess of aquifer recharge such as in Arizona and Utah. An earth fissure generally exhibits characteristics including orientation, discrete rupture, local range and long extension, although horizontal displacements across earth fissures are relatively small. In China, the frequency and magnitude of earth fissures is much greater and has caused associated hazards. For example, the earth fissures have damaged infrastructures including roads, gas lines and buildings, which has presented a danger to the public. Many experts have worked on measurement methods, mechanism analysis, risk assessment, modeling and forecasting, as well as mitigation methods of earth fissures. Visualization of fissures is a perquisite to these studies.

Three-dimensional (3-D) visualization in hydrogeology has been frequently used for inspecting field data and for understanding model results (Voss, 1999). Compared to numer- 
ical values, 3-D visualization is sometimes more effective for researchers to learn the distribution of geologic phenomena in 3-D domains. Generally there are two methods including the surface and solid methods for displaying the geological structure. Surface methods such as multiple DEM (digital elevation model) are popular and easier to be realized, while, the model from this method can not reflect the inner materials or attributs in geologic structures. Solid methods can overcome this shortcoming and models from this method are realized in three dimensions. Generalized Tri-Prism (GTP) was proposed for the layered strata (Wu, 2004), which can be used to simulate complicated geological structures. There are few studies on the visualization of earth fissures. Earth fissures are generally shown by photos or schematic drawings. Zhu et al. (2007) used the polygon of earth fissure to rebuild the earth surface model in the Suzhou-Wuxi-Changzhou area (short for Su-Xi-Chang). Li et al. (2010) expressed ground fissures' spatial continuous curved surfaces by using the linear-distributed ground fissures data and geological model with a surface method of Triangle Irregular Networks (TIN) in Fenwei Plain. However, geologic models in these studies are quasi-3-D.

OpenGL is a 3-D graphics software library developed by SGI Inc. (Silicon Graphics). OpenGL is composed of hundreds of commands and functions for creating real-time 3-D graphics, which can be used to generate 3-D object with high fidelity, and can realize the interactive operations with the simulated objections. To enhance the fidelity, lighting conditions including ambient illumination and diffuse light are defined to create a 3-D scene.

This paper aims to investigate a 3-D visualization method to exhibit the distribution of earth fissures. Yingguoan in Su$\mathrm{Xi}$-Chang was taken as an example area. Visual $\mathrm{C}++$ was used as a developed platform and OpenGL was employed to create real-time 3-D graphics for the visualization of a geologic model with earth fissures.

\section{Description of case study area}

There are numerous earth fissures in the Su-Xi-Chang area, Jiangsu Province, China, which lies in the alluvial plain of the Yangtze River Delta. The earth fissures that developed in 1991 in Yinguoan are typical and ere induced by steep bedrock surfaces and differential subsidence. There is a fracture belt extending $2000 \mathrm{~m}$ and $50-150 \mathrm{~mm}$ width along the NE direction, which shows obvious tensile characteristics (Yu et al., 2004, 2013). A 3-D seismic exploration, covering a survey area of $1.7 \mathrm{~km}^{2}$, was carried out to detect the earth fissures in this area. These seismic data were interpreted to extract the detailed information on stratagraphy and the 3-D shape of earth fissures, such as width, depth and length on the basis of the seismic velocity and logging.

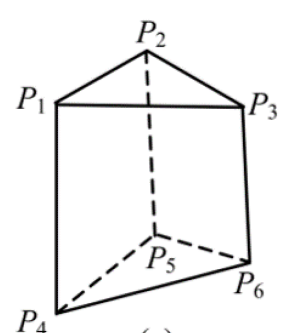

(a)

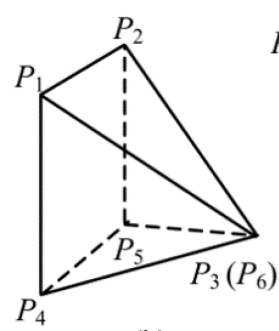

(b)

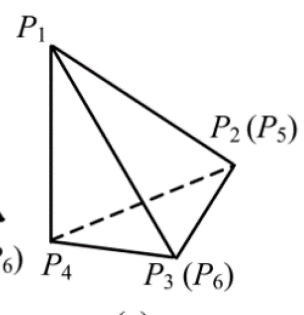

(c)
Figure 1. Different GTP elements. (a) The up surface and the down surface of GTP are not necessarily parallel. (b) The edge of P3 and P6 converges to one point. (c) The edge of P2 and P5 also converges to another point.

\section{Methodology}

Detailed information of Aquifer II was interpreted from 3D seismic data. Scattered points of six layers were obtained. Each point has a lithologic description and coordinates $(X$, $Y, Z$ ). Information of earth fissures includes the outlines of the fissures on the ground, extension and dip. The earth fissures are assumed to be a "V" shaped.

The detailed process of visualizing the earth fissures using the GTP method is as follows.

Firstly, a geologic model without fissures was constructed. The up surface and the down surface of GTP are not necessarily parallel (Fig. 1a) and there are other special shapes. For example, the edge of $P_{3}$ and $P_{6}$ converges to one point (Fig. 1b) and $P_{2}$ and $\mathrm{P}_{5}$ also to another point (Fig. 1c), which can simulate complicated geological structures such as faults and stratagraphic pinch outs. There are six points in each GTP stored in the database, which is easy for query. For example, $P_{3}$ and $P_{6}$ (Fig. 1b, c) are stored separately but with the same coordinates. The up and down surfaces of each GTP, defined by Triangle Irregular Networks such as $P_{1} P_{2} P_{3}$ and $P_{4} P_{5} P_{6}$, consist of the surface of each stratum. The inner solid between two surfaces are of series of triangular prisms. These triangular prisms can further be split.

Secondly, an earth fissure model was built. An earth fissure is a polygon on the ground and the width decreases linearly with depth. The vertices of the polygon are assumed to be symmetrical. The earth fissure volume was constructed by a series of triangular pyramids.

Thirdly, a geological model with earth fissures was developed. The geologic model and the earth fissures model were combined using Boolean operators. Three types of cutting were involved in the process. They are whole cutting, special cutting and false cutting (Li et al., 2008). No cutting will be carried out if six points of GTP are at the same side of the cutting plane. The cutting type is determined by the intersection points of the cutting plane and the GTP. Then the origin GTP is re-build and the topology relationship of GTP is renewed. 

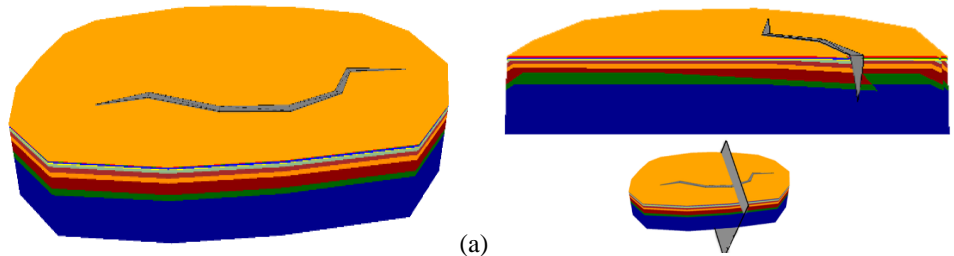

(b)

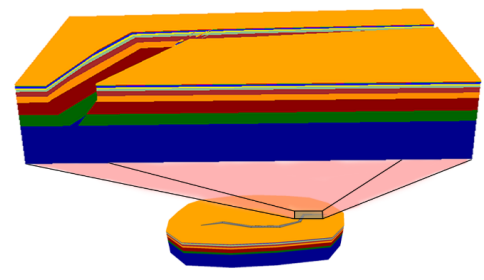

(c)

Figure 2. Geologic structure with earth fissure model. (a) The geological body with earth fissures model. (b) The spatial relations between the strata and the fissures. (c) The combination of bottom part of (b) and a close-up of the model.

\section{Results and conclusions}

GTP represents the element for visualization. The top and bottom triangular surfaces, and the lateral quadrilateral are drawn by using the OpenGL functions of glBegin(GL_TRIANGLES) and glBegin(GL_QUADS). The modeling results can be displayed as the "skeleton" pattern by using wireframe in OpenGL. At the solid mode, each surface of GTP with different materials in the geological model can be specified with different colors. The earth fissures are plotted as a series of triangles in grey. The geological body with earth fissures model (Fig. 2a) can be clipped with any line, which is convenient for researchers to learn the spatial relations between the strata and the fissures (Fig. 2b). The model can also be zoomed in and out (Fig. 2c).

This paper presents a process to visualize the geological model with earth fissures based on the GTP method. The geological model can be subdivided by a fissure model to derive the geological structure with each earth fissure. We can set attributes such as permeability or storage to each GTP element, which is in a real 3-D domain. This method can simulate complicated geological phenomena. In this study, the earth fissures are assumed to be "V" shaped, while, the real distribution of fissures is more complex.

Acknowledgements. This study is funded by National Natural Science (No. 41201420, 41130744) and Key Laboratory of Earth Fissures Geological Disaster, Ministry of Land and Resources, Geological Survey of Jiangsu Province, Nanjing.

\section{References}

Li, B., Zhang, J., Xu, Y., Lv, Y., Zhang, L., Wang, W., Yan, F., $\mathrm{Xu}$, M., Xing, C., and Wang, S.: Study on modeling and 3-Dvisualization of ground fissures' spatial curved surfaces, Image and Signal Processing (CISP), 6, 2838-2844, 2010.

Li, C.-C., Wang, B.-S., and Xue, H.-Z.: Coal geological body threedimensional modeling and cutting based on GTP, Journal of China Coal Society, 33, 1268-1271, 2008 (in Chinese).

Wu, L.: Topological relations embodied in a generalized tri-prism (GTP) model for a 3-D geosciences modeling system, Comput. Geosci., 30, 405-418, 2004.

Yu, J., Wang, X. M., Su, X. S., and Yu, Q.: The mechanism analysis on ground fissure disaster formation in Suzhou-Wuxi-Changzhou area, Journal of Jilin University, Earth Science Edition, 34, 236 241, 2004 (in Chinese).

Yu, J., Su, X., Zhu, L., Duan, F.-Z., Pan, Y., Gao, L., and Wu, S.-L.: Research on 3-D visualized strata model virtual reality system of land subsidence in Suzhou-Wuxi-Changzhou area, 8th International Symposium on Land Subsidence, 17-22 October 2010, Queretaro, Mexico, 339, 108-113, 2010

Yu, J., Zhu, J. Q., Gong, X. L., and Yang, Y.: The potential mechanisms of Yingguoan earth fissure using 3D seismic exploration data, 20th International Congress on Modelling and Simulation, 1-6 December 2013, Adelaide, Australia, 2751-2757, 2013.

Voss, C.: Editor's Message: Scientific visualization in hydrogeology, Hydrogeol. J., 7, 153-154, 1999.

Zhu, L., Su, X., Lin, X., Gong, H., and Yu, J.: Application of VRGIS in studying ground fracture in the western region of Wuxi area, Journal of Jilin University, Earth Science Edition, 2, 317 321, 2009 (in Chinese). 